\title{
Movement Simulation of Automatic Transmission Gearbox based on EON Studio
}

\author{
Kelbesa Edosa Ketema ${ }^{1}$, Guo Qin Li $^{2}$, Xi Ping Song ${ }^{3}$ \\ ${ }^{1}$ Tianjin University of Technology and Education, Tianjin, 300222, China
}

\begin{abstract}
Automatic Transmission Gearbox has many moving parts all with a special functions. To operate the machine you should understand the names and their movements. The Movement Simulation of Automatic Transmission Gearbox is developed by virtual reality development platform of EON Studio. A 3D model of Automatic Transmission Gearbox is built through $3 D$ realistic modeling tools of solidworks software, and transformed into EON Studio..
\end{abstract}

Keywords: Virtual reality, Movement simulation, Automatic Transmission Gearbox, EON studio, Solidwork software

\section{Introduction}

Nowadays, the virtual reality technology provides an interesting way to experiment a great variety of situations, these can go from scientific simulation, passing through learning environments, up to the most unimaginable virtual worlds run to the entertainment. A virtual simulation is an effective way to allow people learn better and train more. This paper presents the Movement simulation of Automatic Transmission Gearbox based on EON Studio.EON Studio is a complete GUI based authoring tool for developing real-time 3D simulations focused on Sales/Marketing, Training, and Visualization . Building EON simulations is like building electronics hardware by connecting existing nodes one to another. The nodes contribute varying effects into a simulation depending on the node's type, the values in its data fields, and the way it is connected to other nodes.

\section{Development of Virtual Automatic Transmission Gearbox}

To simulate a physical scenario you will need to create 3D model which represents the real physical object and then animate the model using computer-based authoring tools specifically designed for this purpose. Fig. 1 shows the steps of developing a virtual environment.

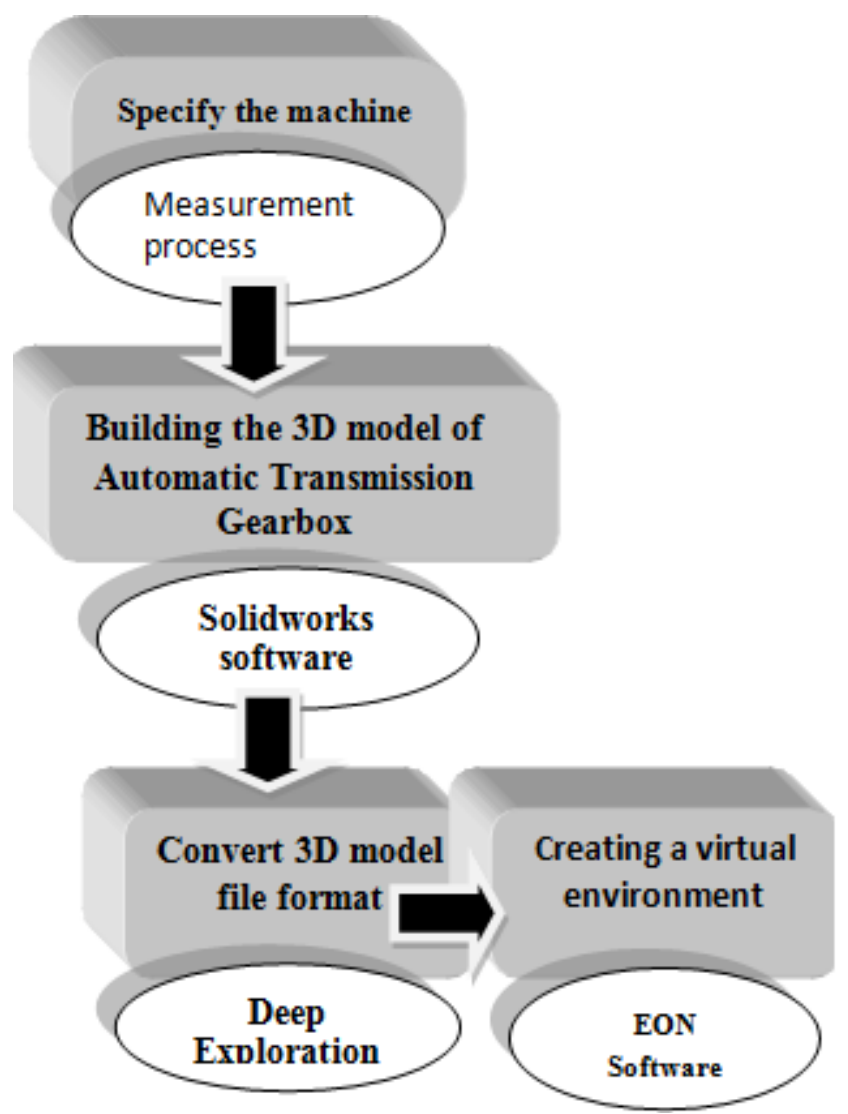

Figure 1: Steps of developing a virtual environment

\section{Building the 3D Model of Automatic Transmission Gearbox}

The paper adopts Automatic Transmission Gearbox model (BMW ZF 6HP26). Shown in Fig.2 as a prototype and uses solidworks software as a modeling tool to construct the 3D realistic model. The modeling process includes whole parts of the machine. Fig.3 shows the 3D model of Automatic Transmission Gearbox built in solidworks. 


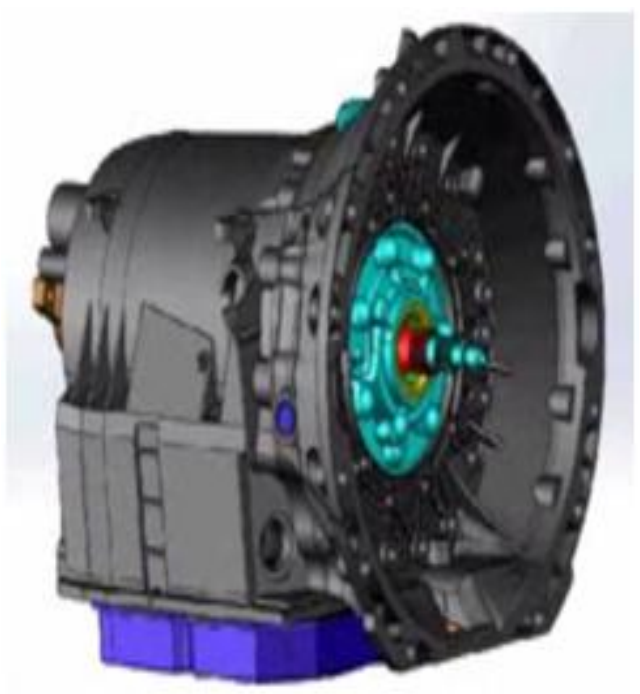

Figure 2: The machine prototype

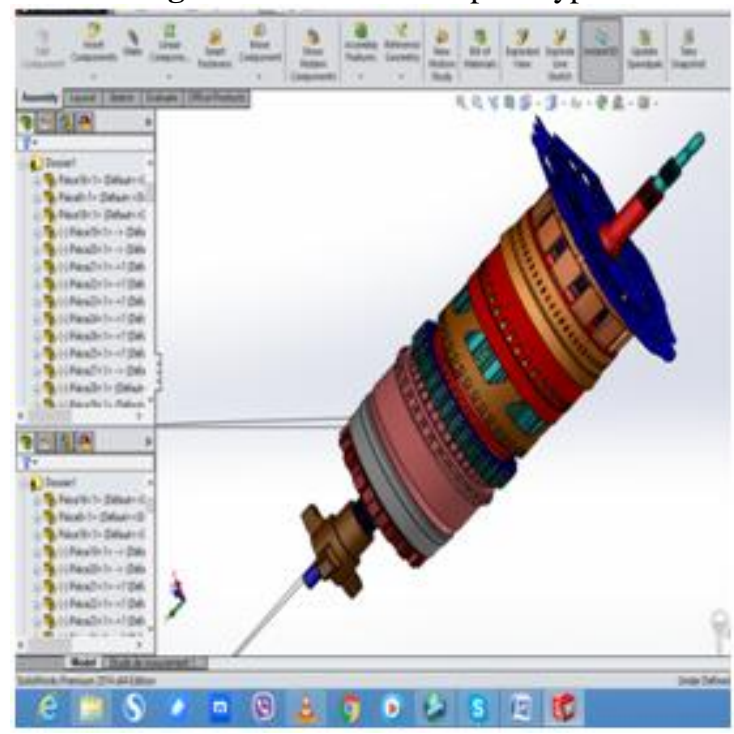

Figure 3: The 3D model of Automatic Transmission Gearbox

\section{Creating a virtual environment}

Before creating the virtual environment we need to convert the 3D model file format from Sldasm format to wrl format by using EONCAD software and Deep Exploration Software. Then transform the 3D model into virtual environment created by EON Studio as shown in Fig.4. After the 3D model was imported into EON Studio we need to rearrange the components in the simulation tree, all the components that moves in the same direction should be placed under the same frame. For example the whole components of the gearbox shaft should be under the same frame as shown in Fig. 5.

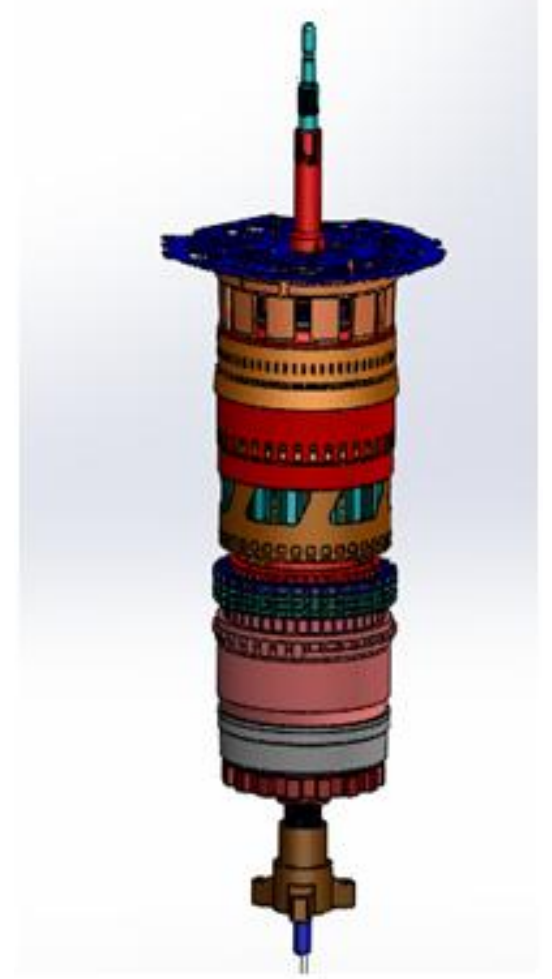

Figure 4: The 3D model in virtual environment

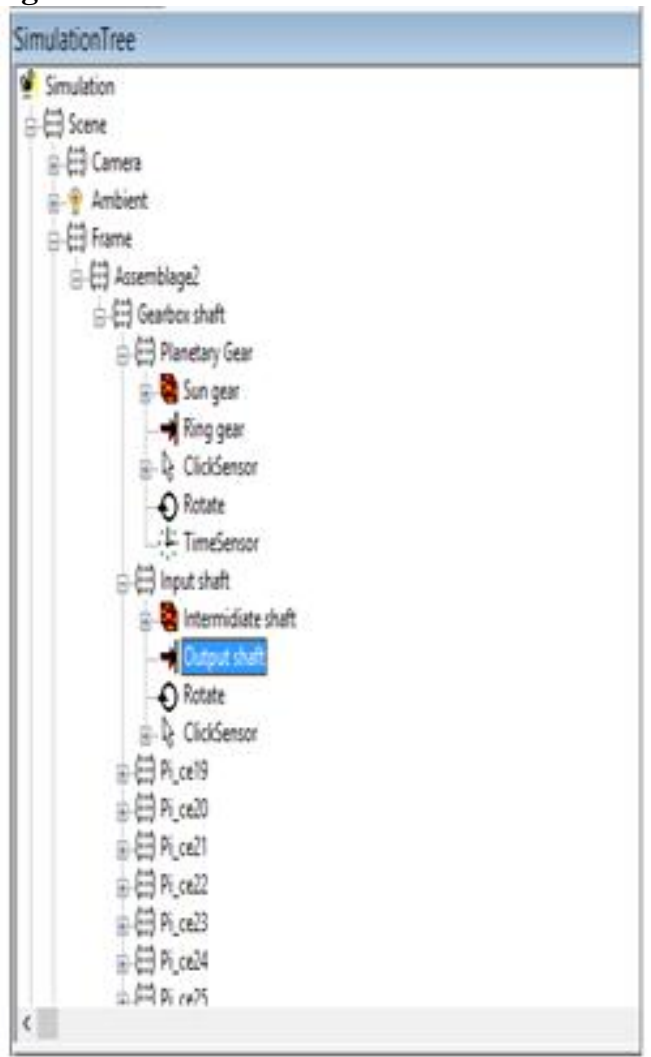

Figure 5: The components in the simulation tree

\section{Simulation Implementation}

To learn the operation of the Automatic Transmission Gearbox, you must be familiar with the names and functions of the principal parts. The simulation is built to simulate the main moving parts of the Gearbox. The predominant form of automatic transmission is hydraulically operated; using a fluid coupling or torque converter, and a set of planetary 


\section{International Journal of Science and Research (IJSR) \\ ISSN (Online): 2319-7064}

Index Copernicus Value (2013): 6.14 | Impact Factor (2015): 6.391

gear sets to provide a range of gear ratios. Major stages of a hydraulic automatic transmission: the torque converter (left), the planetary gear sets and clutch plates (center), as well as hydraulic and electronic controls (bottom). In this section we illustrate the simulation implementation of the shafts and gear movement. Motion node and place node are used to apply movement as shown simulation tree.

By selecting a Node or Prototype in the Component Window and pressing the F1 key, detailed information about how it works will show up in a pop-up window (EON Help). Select the Prototype tab in the lower left corner of the Component window and drag the ObjectNavLITE Prototype to Scene in the Simulation tree. The easiest way to find a Node or a Prototype in the Component window is to type in the first letter of the node name in the text window at the top of the Component window. Delete the Walk node from Scene/Camera in the Simulation Tree. Open the simulation. Please see images below how to control the navigation. Navigate using left (rotate), right (zoom) or pan (both left and right) until you have he view that you want to have when you restart the simulation, press the Initial View button next to the selection tools. Close the simulation window. Re-open the simulation window and see if you have the initial view that you want. If not, move to a new camera position and press the button before closing the window.

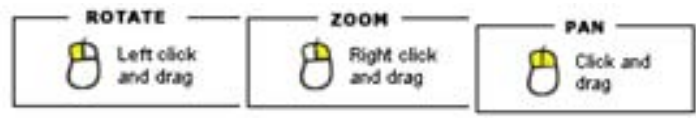

Drag and drop nodes from component window to simulation tree.

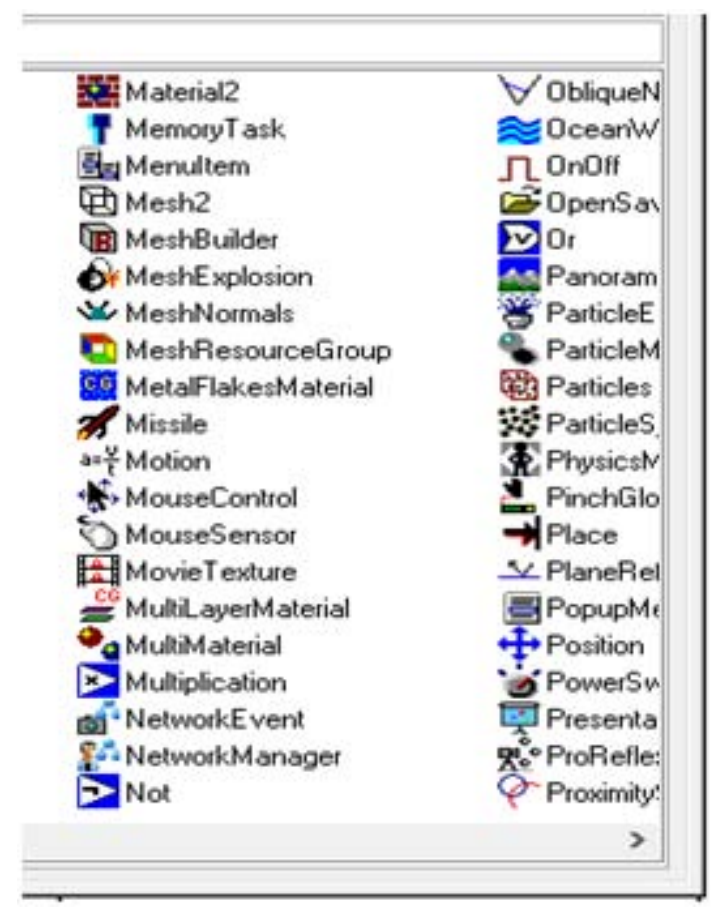

Figure 6: nodes in component window

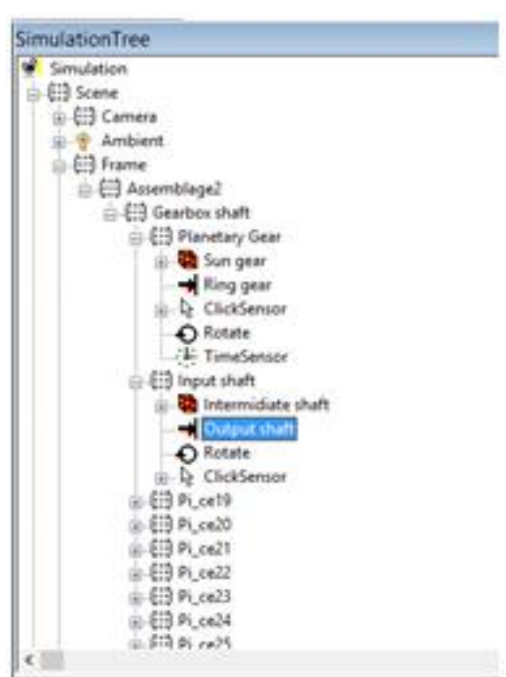

Figure 7: Nodes in simulation tree

Table 1: The relations between nodes

\begin{tabular}{|c|c|c|c|}
\hline Source node & Out-event & $\begin{array}{c}\text { Destination } \\
\text { node }\end{array}$ & In-event \\
\hline ClickSensor & OnButtonDownTrue & Plac169 & SetRun \\
\hline Place171 & stopped & Place168 & Start \\
\hline Place162 & stopped & Place157 & Start \\
\hline Place158 & Stopped & Place163 & Start \\
\hline Place166 & Stopped & Place134 & Start \\
\hline Place156 & Stopped & Place159 & Start \\
\hline
\end{tabular}

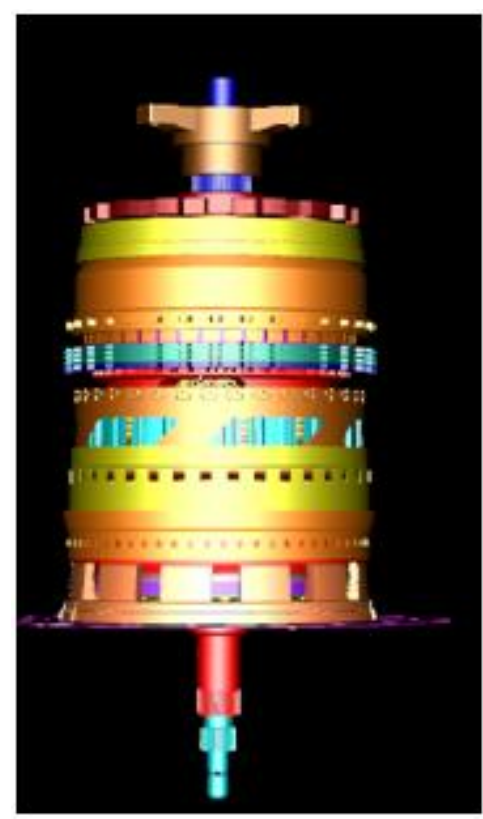

Figure 9: 3D in EON Studio 


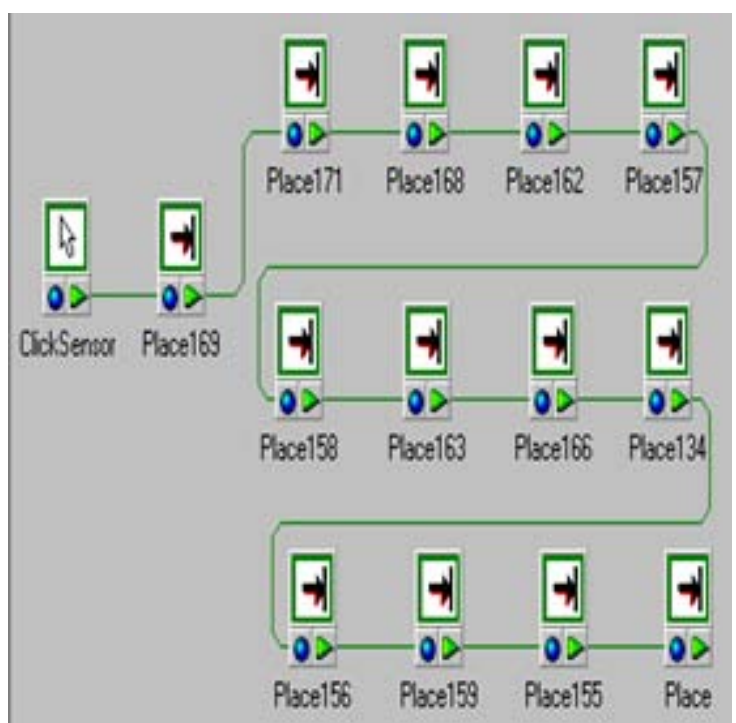

Figure 8: Nodes connected in route simulation

\section{Results}

The proper connections ensure that users can control the parts movement by pressing the corresponding key. When the user pressing the left or right key the parts will moving along the ways to simulate the desired movement as some parts shown in Fig.10 below

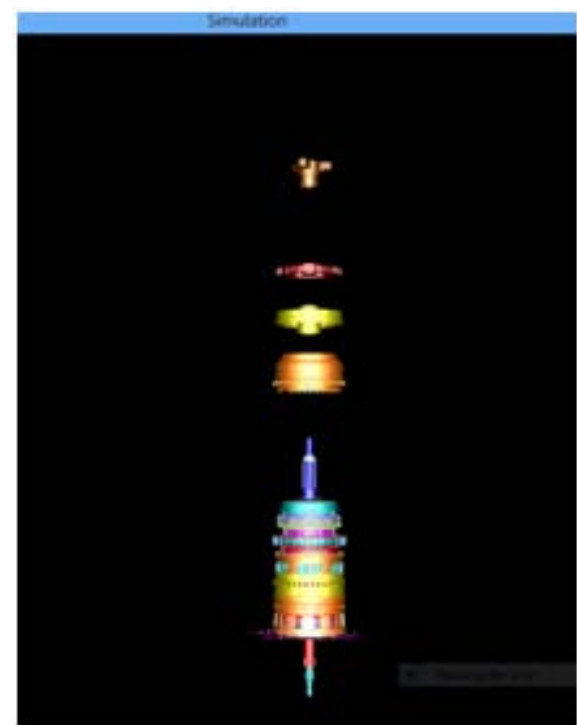

Figure 10: The result of the implementing movement by using nodes to disassemble parts

\section{Conclusions}

The simulation allows users to interact and discover the movement of Automatic Transmission gearbox parts. Virtual environment was developed in EON studio software, it is a powerful tool used for building virtual simulations. The movement of the machine parts was implemented by connecting the appropriate node function.

\section{References}

[1] Jump up^ ${ }^{\wedge}$ Warwick, Alan. "Who Invented the Automatic Gearbox". North West Transmissions Ltd. Retrieved 11 October 2014.
[2] Justice · Bergerud , Garrison, Cafiero, Churches, Interactive $3 \mathrm{D}$ application development Using EON professional for creating 3D visualizations, EON Reality, Inc. and the Kentucky Community and Technical College System, 2010.

[3] Information on www.eonreality.com, AN EON REALITY WHITE PAPER, FEBRUARY, 1999

[4] Information on http://www.eonreality.com/support

[5] X. Daniel Fang, S. Luo, N. J. Lee, and F. Jin, Virtual machining lab for knowledge learning and skills training, Comput Appl Eng Educ 6 (1998), 8997.

[6] Design practice: passanger car automatic transmission, third edition AE-18, Society of Automotive Engineers, Inc, USA, 1994

[7] Biocca, Frank. (1992). Virtual reality technology: A tutorial. Journal of Communication. 42, (4, autumn), 23 72. 